\title{
Nonlogarithmic Repulsion of Transmission Eigenvalues in a Disordered Wire
}

\author{
C. W. J. Beenakker and B. Rejaei \\ Instituut-Lorentz, University of Leiden, P.O. Box 9506, 2300 RA Leiden, The Netherlands
}

(Received 2 July 1993)

\begin{abstract}
An exact solution is presented of the Fokker-Planck equation which governs the evolution of an ensemble of disordered metal wires of increasing length, in a magnetic field By a mapping onto a free-fermion problem, the complete probability distribution function of the transmission elgenvalues is obtained. The logarithmic eigenvalue repulsion of random-matrix theory is shown to break down for transmission eigenvalues which are not close to unity.

PACS numbers: $72.10 . \mathrm{Bg}, 05.60 .+\mathrm{w}, 72.15 . \mathrm{Rn}, 73.50 . \mathrm{Bk}$
\end{abstract}

Level repulsion is the phenomenon that the eigenvalues of a large Hermitian matrix with randomly chosen elements have a small probability for close separation. The importance of this mathematical fact for the physical properties of a complex quantum mechanical system was first noticed in 1955 by Landau and Smorodinsky [1]. In the absence of correlations among the matrix elements, the probability for close separation of two eigenvalues $E$ and $E^{\prime}$ vanishes as $\left|E^{\prime}-E\right|^{\beta}$. (The number $\beta$ equals 1 in a zero magnetic field and 2 in a timereversal-symmetry breaking magnetic field, while $\beta=4$ in zero field with strong spin-orbit scattering [2].) Mathematically, level repulsion originates from the Jacobian $J=\prod_{\imath<\jmath}\left|E_{\jmath}-E_{\imath}\right|^{\beta}$ of the transformation from matrix space to eigenvalue space. Wigner [3] introduced the notion of level repulsion as a "force" by interpreting the Jacobian as a Boltzmann weight, $J=e^{-\beta W}$, with $W=-\sum_{\imath<\jmath} \ln \left|E_{\jmath}-E_{\imath}\right|$. This interpretation of the energy spectrum as a one-dimensional gas of logarithmically repelling classical particles in equilibrium at temperature $\beta^{-1}$ is the essence of the Wigner-Dyson random-matrix theory.

The analog of level repulsion for transmission eigenvalues formed the basis of Imry's 1986 theory of universal conductance fluctuations (UCF) [4]. (The transmission eigenvalues $T_{n}, n=1,2, \ldots, N$, are the eigenvalues of the matrix product $t t^{\dagger}$, with $t$ the $N \times N$ transmission matrix of the conductor and $N$ the number of scattering channels at the Fermi level.) By computing the Jacobian from the space of scattering matrices to the space of transmission eigenvalues, Muttalib, Pichard, and Stone [5] formulated a random-matrix theory (RMT) of quantum transport, along the lines of the Wigner-Dyson RMT of energy levels. This new Jacobian $J=\prod_{\imath<3}\left|\lambda_{3}-\lambda_{\imath}\right|^{\beta}$ takes the same form as for energy levels in terms of the ratio $\lambda_{n} \equiv\left(1-T_{n}\right) / T_{n}$ of reflection to transmission eigenvalues. By postulating that all correlations among the transmission eigenvalues are due to the Jacobian, one arrives at a probability distribution $P$ of the form $P=J \prod_{\imath} f\left(\lambda_{2}\right)$. All microscopic parameters (sample length $L$ and width $W$, mean free path $l$ ) are contained in the single function $f(\lambda)$. This strong assumption could be justified by a "maximum entropy principle" in the quasi-one-dimensional (quasi-1D) limit $L \gg W$ of a wire which is much longer than wide [5-7]. As in the case of the energy levels, $P$ can be written as a Boltzmann weight,

$$
\begin{aligned}
& P=e^{-\beta W}, W=\sum_{\imath<\jmath} u\left(\lambda_{\imath}, \lambda_{\jmath}\right)+\sum_{\imath} V\left(\lambda_{\imath}\right), \\
& u\left(\lambda_{\imath}, \lambda_{\jmath}\right)=-\ln \left|\lambda_{\jmath}-\lambda_{\imath}\right|,
\end{aligned}
$$

with $V=-\beta^{-1} \ln f$ playing the role of a confining potential.

It was originally believed that the distribution function (1) was in exact agreement with the diagrammatic perturbation theory of UCF [8], which for a quasi-1D conductor yields a variance

$$
\operatorname{Var} G / G_{0}=\frac{2}{15} \beta^{-1}
$$

for the sample-dependent fluctuations of the conductance $G$ (in units of $G_{0} \equiv 2 e^{2} / h$ ). However, recently it was calculated [9] that Eq. (1) yields a coefficient $\frac{1}{8}$ instead of $\frac{2}{15}$ in Eq. (2) independently of the form of $V(\lambda)$. The difference between $\frac{1}{8}$ and $\frac{2}{15}$ is tiny, but it has the fundamental implication that the interaction between the $\lambda$ 's is not precisely logarithmic, or in other words, that there exist correlations between the transmission eigenvalues beyond those induced by the Jacobian. What then is the correct distribution function? Is it still of the form (1) but with a nonlogarithmic $u\left(\lambda_{2}, \lambda_{3}\right)$ ? Or is there a many-body interaction $u\left(\lambda_{1}, \lambda_{2}, \ldots, \lambda_{N}\right)$ which cannot be reduced to the sum of pair interactions? That is the problem addressed in this paper.

Our analysis is based on the Dorokhov-Mello-PereyraKumar equation [10] for the evolution of the eigenvalue distribution function in the ensemble of disordered wires of increasing length,

$$
l \frac{\partial P}{\partial L}=\frac{2}{\gamma} \sum_{\imath=1}^{N} \frac{\partial}{\partial \lambda_{\imath}} \lambda_{\imath}\left(1+\lambda_{\imath}\right) J \frac{\partial}{\partial \lambda_{\imath}} J^{-1} P,
$$

where $\gamma \equiv \beta N+2-\beta$. Equation (3) has to be solved for the ballistic initial condition $\lim _{L \rightarrow 0} P=\prod_{2} \delta\left(\lambda_{2}\right)$. The complicated differential operator on the right-hand side (rhs) of Eq. (3) is the Laplacian (Laplace-Beltrami 
operator) in the Riemannian space of transmission eigenvalues [11]. An essentially equivalent "supersymmetry formulation" of the diffusion process described by Eq. (3) has been given by Iida, Weidenmüller, and Zuk [12]. The significance of Eq. (3) is that it satisfies a central limit theorem for multiplication of isotropic transfer matrices $[6,13]$. The isotropy condition restricts its validity to quasi-1D geometries $L \gg W$. Mello and Stone [14] have shown that for these geometries Eq. (3) yields results for the average conductance and its fluctuations in precise agreement with diagrammatic perturbation theory.

Because of the strong coupling between the $N$ degrees of freedom, it has so far only been possible to compute from $\mathrm{Eq}$. (3) the first two moments of the conductance $[14,15]$. This is not sufficient to determine the form of the eigenvalue interaction, which requires knowledge of the complete distribution function. Previous work in this direction was restricted to the case $N=1$ of a single degree of freedom [16]. Here we wish to announce that we have succeeded in solving Eq. (3) exactly for $\beta=2$ and arbitrary $N$.

The solution proceeds in four steps. The first step is to transform from the transmission eigenvalues $T_{n}$ to a new set of variables $x_{n}$, defined by

$$
T_{n} \equiv 1 / \cosh ^{2} x_{n}
$$

The physical significance of the $x$ variables is that $L / x_{n}$ equals the channel-dependent localization length of the conductor [6]. Since $T_{n} \in[0,1], x_{n} \in[0, \infty)$. Substituting $\lambda_{n} \equiv \sinh ^{2} x_{n}$, one finds from Eq. (3) that the probability distribution of the $x$ variables satisfies a FokkerPlanck equation with constant diffusion coefficient,

$$
\begin{aligned}
& \frac{\partial}{\partial s} P\left(\left\{x_{n}\right\}, s\right)=\frac{1}{2 \gamma} \sum_{\imath=1}^{N} \frac{\partial}{\partial x_{\imath}}\left(\frac{\partial P}{\partial x_{\imath}}+\beta P \frac{\partial \Omega}{\partial x_{\imath}}\right) \\
& \Omega=-\sum_{\imath<\jmath} \ln \left|\sinh ^{2} x_{\jmath}-\sinh ^{2} x_{\imath}\right|-\frac{1}{\beta} \sum_{\imath} \ln \left(\sinh 2 x_{\imath}\right),
\end{aligned}
$$

where we have defined $s \equiv L / l$.

The second step is to map the Fokker-Planck equation (5) onto a Schrödinger equation by means of the substitution

$$
P\left(\left\{x_{n}\right\}, s\right)=e^{-\frac{1}{2} \beta \Omega\left(\left\{x_{n}\right\}\right)} \Psi\left(\left\{x_{n}\right\}, s\right) .
$$

This is a variation on Sutherland's transformation [17], which we used in Ref. [18] in a different context. Substitution of Eq. (6) into Eq. (5) yields for $\Psi$ a Schrödinger equation in imaginary time,

$$
\begin{gathered}
-\frac{\partial \Psi}{\partial s}=(\mathcal{H}-U) \Psi \\
\mathcal{H}=-\frac{1}{2 \gamma} \sum_{\imath}\left(\frac{\partial^{2}}{\partial x_{\imath}^{2}}+\frac{1}{\sinh ^{2} 2 x_{\imath}}\right) \\
+\frac{\beta(\beta-2)}{2 \gamma} \sum_{\imath<\jmath} \frac{\sinh ^{2} 2 x_{\jmath}+\sinh ^{2} 2 x_{\imath}}{\left(\cosh 2 x_{\jmath}-\cosh 2 x_{\imath}\right)^{2}}
\end{gathered}
$$

$U=-\frac{N}{2 \gamma}-N(N-1) \frac{\beta}{\gamma}-N(N-1)(N-2) \frac{\beta^{2}}{6 \gamma}$

For a particular ordering of the $x_{n}$ 's, the function $\Psi_{0} \propto \exp \left(-\frac{1}{2} \beta \Omega\right)$ is an eigenfunction of the $N$-fermion Hamiltonian $\mathcal{H}$ with eigenvalue $U$ [since $e^{-\beta \Omega}$ is an $s$ independent solution of Eq. (5)]. Antisymmetrization yields the fermion eigenstate

$$
\Psi_{0}\left(\left\{x_{n}\right\}\right)=C e^{-\frac{1}{2} \beta \Omega\left(\left\{x_{n}\right\}\right)} \prod_{\imath<j} \frac{x_{\jmath}-x_{\imath}}{\left|x_{\jmath}-x_{\imath}\right|},
$$

with $C$ a normalization constant.

The third step is to relate the $N$-fermion Green's function $G\left(\left\{x_{n}\right\}, s \mid\left\{y_{n}\right\}\right)$ of the Schrödinger equation (7) to the solution $P\left(\left\{x_{n}\right\}, s \mid\left\{y_{n}\right\}\right)$ of the Fokker-Planck equation (5) with symmetrized delta-function initial condition

$$
P\left(\left\{x_{n}\right\}, 0 \mid\left\{y_{n}\right\}\right)=\frac{1}{N !} \sum_{\pi} \prod_{\imath=1}^{N} \delta\left(x_{\imath}-y_{\pi_{\imath}}\right) .
$$

The sum in Eq. (11) is over all $N$ ! permutations of $1,2, \ldots, N$. Eventually, we will take the limit $\left\{y_{n}\right\} \rightarrow 0$ of a ballistic initial condition, but to carry out this limit correctly it helps to first consider the more general initial condition (11). The functions $P$ and $G$ are related by a similarity transformation,

$$
P\left(\left\{x_{n}\right\}, s \mid\left\{y_{n}\right\}\right)=\Psi_{0}\left(\left\{x_{n}\right\}\right) G\left(\left\{x_{n}\right\}, s \mid\left\{y_{n}\right\}\right) \Psi_{0}^{-1}\left(\left\{y_{n}\right\}\right) .
$$

For $\beta=2$ the interaction term in Eq. (8) vanishes identically, reducing $\mathcal{H}$ to a sum of single-particle Hamiltonians $\mathcal{H}_{0}$

$$
\mathcal{H}_{0}=-\frac{1}{4 N} \frac{\partial^{2}}{\partial x^{2}}-\frac{1}{4 N \sinh ^{2} 2 x} .
$$

It might be possible to solve also the interacting Schrödinger equation ( 7 ) for $\beta=1$ or 4 , by some modification of techniques developed for the Sutherland Hamiltonian $[17,19]$, but here we will only consider the simplest case $\beta=2$ of broken time-reversal symmetry. The spectrum of $\mathcal{H}_{0}$ is continuous, with eigenvalues $\varepsilon=k^{2} / 4 N$. The (real and normalized) eigenfunctions are

$$
\psi_{k}(x)=\left[\pi k \tanh \left(\frac{1}{2} \pi k\right) \sinh (2 x)\right]^{1 / 2} P_{\frac{1}{2}(\mathrm{i} k-1)}(\cosh 2 x),
$$

where $P_{\nu}(x)$ is a Legendre function. The single-particle Green's function $G_{0}(x, s \mid y)$ is

$$
G_{0}(x, s \mid y)=\int_{0}^{\infty} \frac{d k}{2 \pi} e^{-k^{2} s / 4 N} \psi_{k}(x) \psi_{k}(y)
$$

The $N$-fermion Green's function $G$ is a Slater determinant of the $G_{0}$ 's,

$$
G\left(\left\{x_{n}\right\}, s \mid\left\{y_{n}\right\}\right)=\frac{e^{U s}}{N !} \operatorname{Det} G_{0}\left(x_{n}, s \mid y_{m}\right)
$$

where Det $a_{n m}$ denotes the determinant of the $N \times N$ matrix with elements $a_{n m}$. 
The fourth step is to take the limit $\left\{y_{n}\right\} \rightarrow 0$ of a ballistic initial condition. The zeros of the Slater determinant (16) for $y_{m} \rightarrow 0$ cancel the poles of $\Psi_{0}^{-1}\left(\left\{y_{n}\right\}\right)$ in Eq. (12), as one can see by expanding $G_{0}(x, s \mid y)$ in powers of $y$. We find

$$
\begin{aligned}
\lim _{\left\{y_{n}\right\} \rightarrow 0} P\left(\left\{x_{n}\right\}, s \mid\left\{y_{n}\right\}\right)= & C(s) \prod_{\imath<\jmath}\left(\sinh ^{2} x_{\jmath}-\sinh ^{2} x_{\imath}\right) \prod_{\imath} \sinh 2 x_{\imath} \\
& \times \operatorname{Det}\left(\int_{0}^{\infty} d k e^{-k^{2} s / 4 N} \tanh \left(\frac{1}{2} \pi k\right) k^{2 m-1} P_{\frac{1}{2}(2 k-1)}\left(\cosh 2 x_{n}\right)\right),
\end{aligned}
$$

with $C(s)$ an $x$-independent normalization constant (such that $P$ is normalized to unity).

The solution (17) holds for any $s$ and $N$. It can be simplified in the regime $1 \ll s \ll N$ of a conductor which is long compared to the mean free path $l$ but short compared to the localization length $N l$. This is the metallic regime [20]. The dominant contribution to the integral over $k$ in Eq. (17) then comes from the range $k \gtrsim(N / s)^{1 / 2} \gg$ 1. In this range $\tanh \left(\frac{1}{2} \pi k\right) \rightarrow 1$ and the Legendre function simplifies to a Bessel function, $P_{\frac{1}{2}(\imath k-1)}(\cosh 2 x) \rightarrow$ $(2 x / \sinh 2 x)^{1 / 2} J_{0}(k x)$, provided $x \gg(s / N)^{1 / 2}$. The $k$ integration can now be carried out analytically,

$$
\int_{0}^{\infty} d k e^{-k^{2} s / 4 N} k^{2 m-1} J_{0}\left(k x_{n}\right)=\frac{1}{2}(m-1) !(4 N / s)^{m} e^{-x_{n}^{2} N / s} L_{m-1}\left(x_{n}^{2} N / s\right),
$$

where $L_{p}(x)$ is a Laguerre polynomial. We then apply the determinantal identity

$$
\operatorname{Det} L_{m-1}\left(x_{n}^{2} N / s\right)=c \operatorname{Det} x_{n}^{2(m-1)}=c \prod_{\imath<j}\left(x_{\jmath}^{2}-x_{\imath}^{2}\right),
$$

$$
\begin{aligned}
P\left(\left\{x_{n}\right\}, s\right)= & C(s) \prod_{\imath<\jmath}\left[\left(\sinh ^{2} x_{3}-\sinh ^{2} x_{\imath}\right)\left(x_{\jmath}^{2}-x_{\imath}^{2}\right)\right] \\
& \times \prod_{\imath}\left[e^{-x_{\imath}^{2} N / s}\left(x_{\imath} \sinh 2 x_{\imath}\right)^{1 / 2}\right]
\end{aligned}
$$

with $c$ an $x$-independent number [which can be absorbed in $C(s)$ ]. Collecting results, we find that the general solution (17) simplifies in the metallic regime to

We now transform back from the variables $x_{n}$ to $\lambda_{n} \equiv$ $\left(1-T_{n}\right) / T_{n} \equiv \sinh ^{2} x_{n}$, and write Eq. (19) in the form

$$
\begin{aligned}
P\left(\left\{\lambda_{n}\right\}, s\right) & =C(s) \exp \left[-\beta\left(\sum_{\imath<\jmath} u\left(\lambda_{\imath}, \lambda_{\jmath}\right)+\sum_{i} V\left(\lambda_{\imath}, s\right)\right)\right], \\
u\left(\lambda_{\imath}, \lambda_{\jmath}\right) & =-\frac{1}{2} \ln \left|\lambda_{\jmath}-\lambda_{\imath}\right|-\frac{1}{2} \ln \left|\operatorname{arcsinh}^{2} \lambda_{\jmath}^{1 / 2}-\operatorname{arcsinh}^{2} \lambda_{\imath}^{1 / 2}\right|, \\
V(\lambda, s) & =\frac{N}{2 s} \operatorname{arcsinh}^{2}\left(\lambda^{1 / 2}\right)\left(1+\mathcal{O}\left(N^{-1}\right)\right),
\end{aligned}
$$

with $\beta=2$. Equation (20) is similar to Eq. (1), but differs in the eigenvalue interaction $u$. For $\lambda \ll 1$ (i.e., for $T$ close to unity) $u\left(\lambda_{2}, \lambda_{\jmath}\right) \rightarrow-\ln \left|\lambda_{3}-\lambda_{2}\right|$, so we derive the logarithmic eigenvalue repulsion (1b) for the strongly transmitting scattering channels. However, for $\lambda \approx 1$ the interaction (20b) is nonlogarithmic. For fixed $\lambda_{\imath} \ll 1$, $u\left(\lambda_{2}, \lambda_{\jmath}\right)$ as a function of $\lambda_{j}$ crosses over from $-\ln \left|\lambda_{\jmath}-\lambda_{2}\right|$ to $-\frac{1}{2} \ln \left|\lambda_{3}-\lambda_{2}\right|$ at $\lambda_{3} \approx 1$. This answers the question raised in the introduction: The eigenvalue interaction (20b) is still a two-body interaction, as in Eq. (1b), but it is different for weakly and for strongly transmitting scattering channels. For weakly transmitting channels it is twice as small as predicted by considerations based solely on the Jacobian, which turn out to apply only to the strongly transmitting channels.

The reduced level repulsion for weakly transmitting channels should yield an enhancement of the conductance fluctuations. To check this, we have computed the twopoint correlation function

$$
K\left(x, x^{\prime}\right)=\langle\rho(x)\rangle\left\langle\rho\left(x^{\prime}\right)\right\rangle-\left\langle\rho(x) \rho\left(x^{\prime}\right)\right\rangle
$$

where $\rho(x)=\sum_{\imath} \delta\left(x-x_{2}\right)$ is the eigenvalue den- sity [21] and $\langle\cdots\rangle$ denotes an average with distribution (19). We compute $K\left(x, x^{\prime}\right)$ according to the general method of Ref. [9], by solving the integral equation $-\int_{0}^{\infty} d x^{\prime} \psi\left(x^{\prime}\right) u\left(x, x^{\prime}\right)=\phi(x)$. The solution $\psi(x)=$ $\int_{0}^{\infty} d x^{\prime} \beta K\left(x, x^{\prime}\right) \phi\left(x^{\prime}\right)$ then yields the function $K\left(x, x^{\prime}\right)$ in the large- $N$ limit. (This limit corresponds to the regime of validity of the diagrammatic perturbation theory of UCF [8].) We find

$$
\begin{aligned}
K\left(x, x^{\prime}\right) & =g\left(x-x^{\prime}\right)+g\left(x+x^{\prime}\right), \\
g(x) & =-\frac{1}{\pi^{2}} \int_{0}^{\infty} d k \frac{k \cos k x}{1+\operatorname{cotanh}\left(\frac{1}{2} \pi k\right)} \\
& =\frac{1}{2 \pi^{2}} \operatorname{Re}\left[\left(x+i 0^{+}\right)^{-2}-(x+i \pi)^{-2}\right] .
\end{aligned}
$$

The variance of an observable $A$ of the form $A=\sum_{\imath} a\left(x_{\imath}\right)$ (a so-called linear statistic) is obtained from

$$
\operatorname{Var} A=-\int_{0}^{\infty} d x \int_{0}^{\infty} d x^{\prime} a(x) a\left(x^{\prime}\right) K\left(x, x^{\prime}\right) .
$$

Substituting Eq. (22) we find 


$$
\begin{aligned}
& \operatorname{Var} A=\frac{1}{2 \pi^{2}} \int_{0}^{\infty} d k \frac{k|a(k)|^{2}}{1+\operatorname{cotanh}\left(\frac{1}{2} \pi k\right)}, \\
& a(k)=2 \int_{0}^{\infty} d x a(x) \cos k x .
\end{aligned}
$$

To obtain the variance of the conductance $G / G_{0}=$ $\sum_{2} T_{2}$ we substitute $a(x)=1 / \cosh ^{2} x$, hence $a(k)=$ $\pi k / \sinh \left(\frac{1}{2} \pi k\right)$, hence

$$
\operatorname{Var} G / G_{0}=\frac{1}{2} \int_{0}^{\infty} d k \frac{k^{3} \exp \left(-\frac{1}{2} \pi k\right)}{\sinh \left(\frac{1}{2} \pi k\right)}=\frac{1}{2} \times \frac{2}{15},
$$

in agreement with Eq. (2) for $\beta=2$. In contrast, Eq. (1) gives a smaller coefficient $\frac{1}{8}$ instead of $\frac{2}{15}$. The difference is so small because only the weakly transmitting channels (which contribute little to the conductance) are affected by the nonlogarithmic interaction (20b). In the same way we can compute the variance of other transport properties [22].

In summary, we have shown that the repulsion between transmission eigenvalues in a disordered metal wire is reduced for weakly transmitting scattering channels. Replacement of the logarithmic level repulsion (1b) by the nonlogarithmic interaction (20b) yields conductance fluctuations in precise agreement with diagrammatic perturbation theory. Between $\lambda \ll 1$ and $\lambda \gg 1$ the repulsion is reduced by simply a factor of 2 , suggesting that there might be a symmetry explanation hiding behind the exact solution.

This research was supported in part by the Dutch Science Foundation NWO/FOM.

Note added.-We have learned that Chalker and Macêdo [23] have also obtained the result (22) for the large- $N$ limit of the two-point correlation function in the metallic regime. (The functional form of the level interaction was not obtained.) Their method of solution of Eq. (3) is approximate, but works for all $\beta \in\{1,2,4\}$, while our solution is exact, but restricted to the case $\beta=2$.

[1] L. Landau and Ya. Smorodinsky, reprinted in Statistical Theornes of Spectra: Fluctuations, edited by C. E. Porter (Academic, New York, 1965).

[2] F. J. Dyson, J. Math. Phys. 3, 1199 (1962).

[3] E. P. Wigner, SIAM Review 9, 1 (1967).

[4] Y. Imry, Europhys. Lett. 1, 249 (1986).

[5] K. A. Muttalib, J.-L. Pichard, and A. D. Stone, Phys. Rev. Lett. 59, 2475 (1987).
[6] A. D. Stone, P. A. Mello, K. A. Muttalib, and J.-L. Pichard, in Mesoscopic Phenomena in Solids, edited by B. L. Al'tshuler, P. A. Lee, and R. A. Webb (NorthHolland, Amsterdam, 1991).

[7] An alternative maximum entropy principle has recently been put forward by K. Slevin and T. Nagao, Phys. Rev. Lett. 70, 635 (1993). The resulting distribution function differs from Eq. (1), but does not improve the agreement with Eq. (2) [K. Slevin (private communication)].

[8] B. L. Al'tshuler, Pis'ma Zh. Eksp. Teor. Fiz. 41, 530 (1985) [JETP Lett. 41, 648 (1985)]; P. A. Lee and A. D. Stone, Phys. Rev. Lett. 55, 1622 (1985).

[9] C. W. J. Beenakker, Phys. Rev. Lett. 70, 1155 (1993); Phys. Rev. B 47, 15763 (1993).

[10] O. N. Dorokhov, Pis'ma Zh. Eksp. Teor. Fiz. 36, 259 (1982) [JETP Lett. 36, 318 (1982)]; P. A. Mello, P. Pereyra, and N. Kumar, Ann. Phys. (N.Y.) 181, 290 (1988).

[11] A. Hüffmann, J. Phys. A 23, 5733 (1990).

[12] S. Iida, H. A. Weidenmüller, and J. A. Zuk, Phys. Rev. Lett. 64, 583 (1990).

[13] P. A. Mello and B. Shapiro, Phys. Rev. B 37, 5860 (1988).

[14] P. A. Mello, Phys. Rev. Lett. 60, 1089 (1988); P. A. Mello and A. D. Stone, Phys. Rev. B 44, 3559 (1991).

[15] M. R. Zirnbauer, Phys. Rev. Lett. 69, 1584 (1992).

[16] V. I. Mel'nikov, Fiz. Tverd. Tela 23, 782 (1981) [Sov. Phys. Solid State 23, 444 (1981)]; P. A. Mello, J. Math. Phys. 27, 2876 (1986).

[17] B. Sutherland, Phys. Rev. A 5, 1372 (1972).

[18] C. W. J. Beenakker and B. Rejaei (to be published).

[19] B. D. Simons, P. A. Lee, and B. L. Altshuler, Phys. Rev. Lett. 70, 4122 (1993).

[20] The solution (17) also simplifies in the opposite regime $1 \ll N \ll s$ of a conductor much longer than the localization length. The result is of the form (19), with the replacements $x_{j}^{2}-x_{\imath}^{2} \rightarrow x_{j}-x_{\imath}$ and $x_{\imath} \sinh 2 x_{\imath} \rightarrow$ $x_{\imath}^{2} \sinh 2 x_{2}$. A discussion of this insulating regime will be given elsewhere.

[21] The average density of the $\lambda$ or $x$ variables follows from the eigenvalue interaction $u$ and confining potential $V$ in Eq. (20). To leading order in $N$ the relation is $-\int_{0}^{\infty} d \lambda^{\prime}\left\langle\rho\left(\lambda^{\prime}\right)\right\rangle u\left(\lambda, \lambda^{\prime}\right)=V(\lambda, s)$. The result is $\langle\rho(x)\rangle=N l / L, x \ll L / l$, in agreement with a direct integration of Eq. (3) by P. A. Mello and J.-L. Pichard, Phys. Rev. B 40, 5276 (1989); see also the numerical simulations by K. Slevin, J.-L. Pichard, and K. A. Muttalib, J. Phys. I (France) 3, 1387 (1993).

[22] For the shot noise $A=\sum_{2} T_{2}\left(1-T_{2}\right)$ we find from Eq. (24) $\operatorname{Var} A=\frac{1}{2} \times \frac{46}{2835}$, in agreement with a moment expansion of Eq. (3) by M. J. M. de Jong and C. W. J. Beenakker, Phys. Rev. B 46, 13400 (1992).

[23] J. T. Chalker and A. M. S. Macêdo, Phys. Rev. Lett. 71, 3693 (1993). 